\title{
Plasma copeptin and chronic kidney disease risk in 3 European cohorts from the general population
}

Ray El Boustany, ${ }^{1,2}$ Irina Tasevska, ${ }^{3}$ Esther Meijer, ${ }^{4}$ Lyanne M. Kieneker, ${ }^{4}$ Sofia Enhörning, ${ }^{3}$ Guillaume Lefèvre, ${ }^{5}$ Kamel Mohammedi, ${ }^{1,6}$ Michel Marre, ${ }^{1,6,7}$ Frédéric Fumeron, ${ }^{1,7}$ Beverley Balkau, ${ }^{8,9}$ Nadine Bouby, ${ }^{1,10}$ Lise Bankir, ${ }^{1,11}$ Stephan J.L. Bakker, ${ }^{4}$ Ronan Roussel, ${ }^{1,6,7}$ Olle Melander, ${ }^{3}$ Ron T. Gansevoort, ${ }^{4}$ and Gilberto Velho ${ }^{1}$

IInserm Research Unit 1138, Centre de Recherche des Cordeliers, Paris, France. ²Danone Nutricia Research, Palaiseau, France. ${ }^{3}$ Departments of Internal Medicine and Clinical Sciences, Lund University, Malmö, Sweden. ${ }^{4}$ Department of Internal Medicine, University Medical Center, Division of Nephrology, University of Groningen, Groningen, Netherlands. ${ }^{5}$ Service de Biochimie et Hormonologie, Assistance Publique - Hôpitaux de Paris, Hôpitaux Universitaires Est ParisienTenon, Paris, France. ${ }^{6}$ Department of Diabetology, Endocrinology and Nutrition, DHU Fire, Assistance Publique - Hôpitaux de Paris, Bichat Hospital, Paris, France. ' UFR de Médecine, Université Paris Diderot, Sorbonne Paris Cité, Paris, France. ${ }^{8}$ Inserm Research Unit 1018, Center for Research in Epidemiology and Population Health, Villejuif, France. 'niversité Paris Sud, Villejuif, France. ${ }^{10}$ Université Paris Descartes, Sorbonne Paris Cité, Paris, France. "UPMC University Paris 6, Sorbonne Universités, Paris, France.

Conflict of interest: REB has received a PhD grant partially from Danone Nutricia Research. KM has received honoraria from Novo Nordisk and Sanofi and nonfinancial support from VitalAire, Servier, and Proteor. MM has received honoraria and grants from Abbott, AstraZeneca, Lilly, MSD, Novo Nordisk, Sanofi, and Servier. RR has been a consultant or on the speakers' bureau for AstraZeneca, Boehringer Ingelheim, Janssen, Lilly, Sanofi, MSD, Physiogenex, and Novo Nordisk; has received research funding from Amgen, Sanofi, and Danone Nutricia Research; and nonfinancial support from Thermo Fischer Scientific. OM has received consultancy fees from Danone Nutricia Research and is inventor on a patent on copeptin as predictor of diabetes development (W0 2010049179 A1).

RTC has received a research grant from Thermo Fischer Scientific

Submitted: April 10, 2018

Accepted: May 23, 2018

Published: July 12, 2018

\section{Reference information:}

JCI Insight. 2018;3(13):e121479.

https://doi.org/10.1172/jici.

insight.121479.
BACKGROUND. The prevalence of chronic kidney disease (CKD) is increasing worldwide. The identification of factors contributing to its progression is important for designing preventive measures. Previous studies have suggested that chronically high vasopressin is deleterious to renal function. Here, we evaluated the association of plasma copeptin, a surrogate of vasopressin, with the incidence of CKD in the general population.

METHODS. We studied 3 European cohorts: DESIR ( $n=5,047$; France), MDCS-CC ( $n=3,643$; Sweden), and PREVEND ( $n=7,684$; the Netherlands). Median follow-up was 8.5, 16.5, and 11.3 years, respectively. Pooled data were analyzed at an individual level for 4 endpoints during followup: incidence of stage 3 CKD (estimated glomerular filtration rate [eGFR] $<60 \mathrm{ml} / \mathrm{min} / 1.73 \mathrm{~m}^{2}$ ); the KDICO criterion "certain drop in eGFR"; rapid kidney function decline (eGFR slope steeper than -3 $\mathrm{ml} / \mathrm{min} / 1.73 \mathrm{~m}^{2} / \mathrm{yr}$ ); and incidence of microalbuminuria.

RESULTS. The upper tertile of plasma copeptin was significantly and independently associated with a 49\% higher risk for stage 3 CKD $(P<0.0001)$; a $64 \%$ higher risk for kidney function decline, as defined by the KDICO criterion $(P<0.0001)$; a $79 \%$ higher risk for rapid kidney function decline $(P<$ $0.0001)$; and a $24 \%$ higher risk for microalbuminuria $(P=0.008)$.

CONCLUSIONS. High copeptin levels are associated with the development and the progression of CKD in the general population. Intervention studies are needed to assess the potential beneficial effect on kidney health in the general population of reducing vasopressin secretion or action.

FUNDING. INSERM and Danone Research Centre for Specialized Nutrition.

\section{Introduction}

The prevalence of chronic kidney disease (CKD) is increasing worldwide (1). It affects as many as $10 \%-15 \%$ of the population and is now recognized as a global public health problem (1). CKD is associated with impaired quality of life, reduced life expectancy, and increased risk of end-stage renal disease (ESRD) and severe cardiovascular events (2). The care for patients with CKD improved markedly in the last decades, but there is still an urgent need to identify modifiable factors that are driving the increase of CKD incidence and prevalence.

It is now recognized that adequate hydration is essential to kidney health (3). Recurrent dehydration and heat stress resulting from extreme occupational conditions were shown to cause subclinical kidney inju- 
ry, leading to permanent kidney damage over time (3-5). This clinical condition known as Mesoamerican nephropathy was documented among young agricultural workers in Central America (6). Results from a few observational studies in the general population suggest that high water intake might have beneficial effects on limiting the decline of kidney function over time $(3,7,8)$. Dehydration and a modest elevation of plasma osmolality are major stimuli for vasopressin (or antidiuretic hormone) secretion by the neurohypophysis. Vasopressin is cosecreted into the blood in an equimolar amount with copeptin, the C-terminal portion of the pre-pro-vasopressin peptide. Copeptin is easier to assay (9-11) and is an adequate surrogate of vasopres$\sin (12-14)$. Experimental evidence supports a causal and direct role of vasopressin in the development and aggravation of CKD through V2 receptor activation (15). As vasopressin secretion can be modulated by water intake, and its actions blocked by nonpeptide selective receptor antagonists (vaptans), the vasopressin system could be a potential therapeutic target for the prevention and treatment of $\operatorname{CKD}(15,16)$.

Positive associations of copeptin with markers of kidney function or with kidney function decline were observed in populations with CKD or at high risk of CKD, such as people with diabetes (17-20), patients with autosomal dominant polycystic kidney disease (ADPKD) (21-23), and kidney transplant recipients (24). However, only limited prospective data are available on the association of plasma copeptin with the risk of new-onset $\mathrm{CKD}$ in the general population $(25,26)$. Therefore, we undertook an individual-level pooled analysis of 3 European cohorts from the general population to assess the association of copeptin with the incidence of various kidney function related outcomes. As circulating levels of vasopressin (25) and copeptin $(14,25-29)$ are as much as $50 \%$ higher in men than in women, we also assessed interactions of sex and copeptin in these associations.

\section{Results}

Clinical characteristics at baseline. Flow diagrams of the Data from Epidemiological Study on Insulin Resistance Syndrome (DESIR; France), Malmö Diet and Cancer Study-Cardiovascular Cohort (MDCS-CC; Sweden), and Prevention of Renal and Vascular End-Stage Disease (PREVEND; the Netherlands) cohorts are shown in Figure 1. Unless stated otherwise, results are given for pooled data of the 3 cohorts. Clinical characteristics of participants at baseline by tertiles of plasma copeptin are shown in Table 1. Briefly, people in the upper tertile of plasma copeptin, as compared with those in the lower tertiles, were older; had higher BMI and blood pressure; were more frequently treated with antihypertensive medication, diuretics, or angiotensin-converting enzyme inhibitors/angiotensin receptor blockers (ACE inhibitors/ARBs); and were more likely to be smokers. They also had higher plasma glucose, cholesterol, triglycerides, cystatin C, and creatinine levels and lower estimated glomerular filtration rate (eGFR).

Incidence of stage 3 CKD during follow-up. Progression toward stage $3 \mathrm{CKD}$, defined as eGFR $<60 \mathrm{ml} /$ $\mathrm{min} / 1.73 \mathrm{~m}^{2}$, during follow-up was observed in 1,124 people (8.6\%). Clinical characteristics at baseline of CKD progressors and nonprogressors are shown in Supplemental Table 1 (supplemental material available online with this article; https://doi.org/10.1172/jci.insight.121479DS1). Baseline plasma copeptin concentration was higher in progressors than in nonprogressors. The cumulative incidence of stage $3 \mathrm{CKD}$ during follow-up by tertiles of baseline plasma copeptin was $6.8 \%$ (tertile 1 [T1]), $8.3 \%$ (T2), and 10.9\% (T3) (Figure 2A). Cox proportional hazards regression analyses confirmed the positive association of the upper tertile of plasma copeptin and $\log _{\mathrm{e}}$ [copeptin] with the incidence of CKD in a minimally adjusted model, including as covariates cohort membership, sex, age, and eGFR at baseline (Table 2, model 1; results from individual cohorts are shown in Supplemental Table 2). When adjusted for several potential confounders, the association of plasma copeptin at baseline with the incidence of CKD remained significant (Table 2, model 2). Cox regression analyses were repeated, with quantitative covariates expressed as qualitative dichotomous (below or above the median) variables, with the exception of tertiles of copeptin. Hazard ratios (HRs) for all covariates are shown in Figure 3.

The KDIGO criterion during follow-up. The Kidney Disease: Improving Global Outcomes (KDIGO) criterion "certain drop in eGFR" was fulfilled by 1,233 people (9.1\%) during follow-up. Characteristics at baseline of progressors and nonprogressors during follow-up are shown in Supplemental Table 1. Baseline plasma copeptin concentration was higher in progressors than in nonprogressors. The cumulative incidence of "certain drop in eGFR" during the study by tertiles of plasma copeptin was 7.1\% (T1), 9.4\% (T2), and 10.8\% (T3) (Figure 2B). A logistic regression analysis confirmed the association of the upper tertile of plasma copeptin and $\log _{\mathrm{e}}$ [copeptin] with the KDIGO criterion during follow-up both in minimally and fully adjusted models (Table 2; results from individual cohorts are shown in Supplemental Table 2). Regression analyses were repeated with quantitative covariates expressed as qualitative dichotomous (below or above the median) vari- 


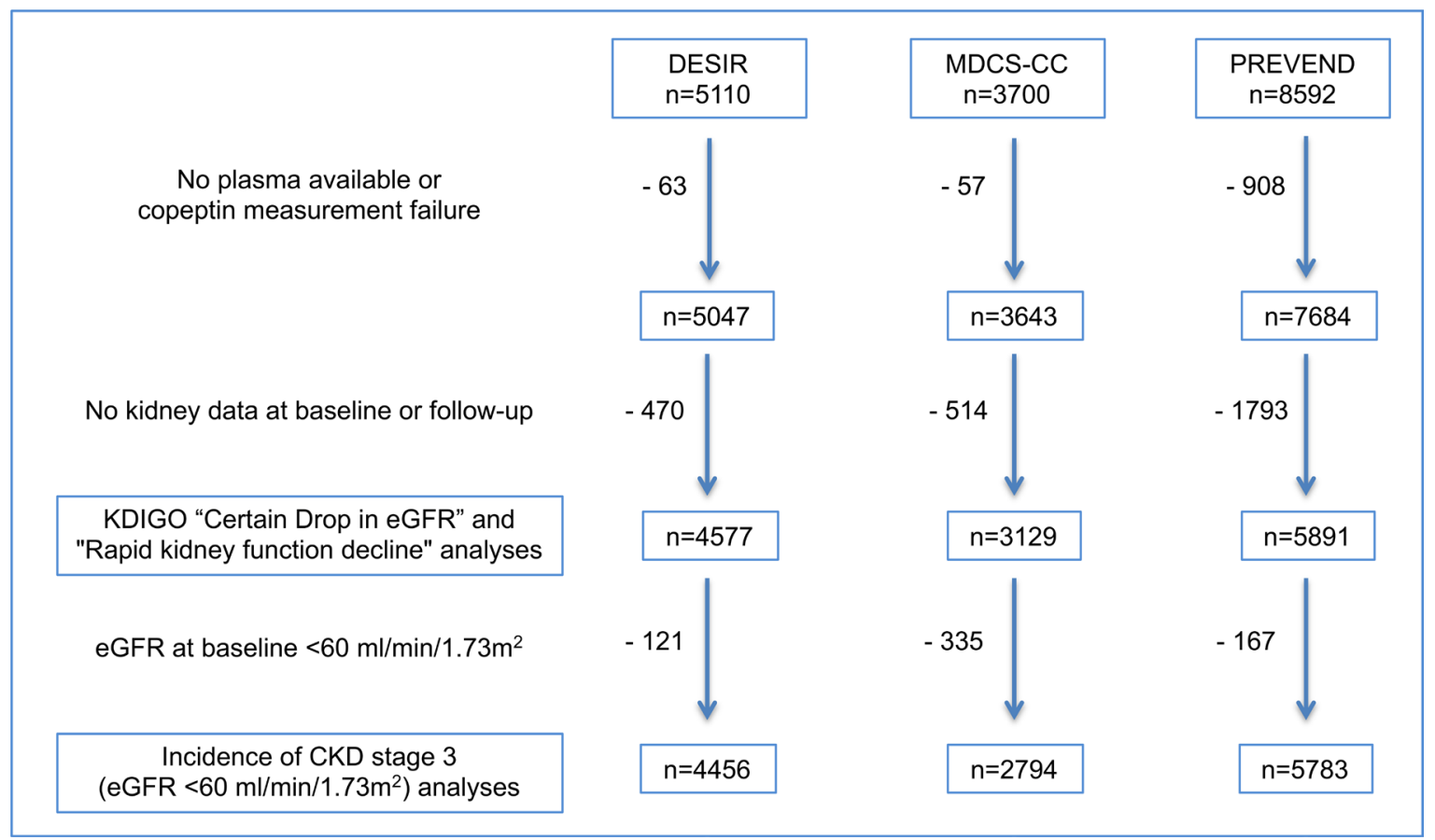

Figure 1. Flow diagram of the participants in the study. Copeptin was measured in 16,374 participants from the DESIR, MDCS-CC, and PREVEND cohorts (women 50.5\%, 59.9\%, and 52.6\%, respectively). People for whom CKD status at baseline or at the end of follow-up could not be ascertained ( $n=2,777$ ) were excluded from the analyses. Thus, analyses were carried out with data from 13,597 participants.

ables, with the exception of tertiles of copeptin. Odds ratio (OR) for all covariates are shown in Figure 3.

eGFR slope and rapid decline in kidney function during follow-up. The rates of change of the eGFR (assessed by the slope) during follow-up by tertiles of plasma copeptin were $-0.65 \pm 0.02$ (T1), $-0.76 \pm 0.02$ (T2), and $-0.79 \pm$ $0.02 \mathrm{ml} / \mathrm{min} / 1.73 \mathrm{~m}^{2}$ per year (T3) (mean $\pm \mathrm{SEM}$, analysis of covariance [ANCOVA], $P<0.0001$, adjusted for age, sex, cohort membership, and eGFR at baseline and duration of follow-up). The difference remained significant in the fully adjusted model $(P<0.0001)$. Rapid kidney function decline (defined by a slope of eGFR steeper than $-3 \mathrm{ml} / \mathrm{min} / 1.73 \mathrm{~m}^{2}$ per year) was observed in 580 people during follow-up. Its prevalence by tertiles of plasma copeptin was 3.2\% (T1), 4.1\% (T2), and 5.5\% (T3) (Figure 2C). A logistic regression analysis confirmed the association of copeptin with rapid kidney function decline during follow-up (Table 2).

Sensitivity analyses - cystatin C-based eGFR. In additional sensitivity analyses in the PREVEND cohort, the eGFR outcomes during follow-up (eGFR $<60 \mathrm{ml} / \mathrm{min} / 1.73 \mathrm{~m}^{2}, \mathrm{KDIGO}$ criterion "certain drop in eGFR," and rapid kidney function decline) were assessed for eGFR computed from serum cystatin $\mathrm{C}$. The upper tertiles of plasma copeptin and $\log _{\mathrm{e}}$ [copeptin] were significantly associated with the outcomes (Supplemental Table 3).

Incidence of albuminuria. The cumulative incidence of albuminuria by tertiles of plasma copeptin was $7.5 \%$ (T1), 7.2\% (T2), and 9.1\% (T3) (DESIR and PREVEND pooled data; Figure 4). Cox proportional hazards regression analyses confirmed the positive association of the upper tertile of plasma copeptin with the incidence of albuminuria: HR 1.24, 95\% CI 1.06-1.46, $P=0.008$ for T3 versus T1/T2, adjusted for cohort, sex, age, systolic and diastolic blood pressure, eGFR, and use of ACE inhibitors or ARBs medication at baseline. The association remained significant when further adjusted for baseline albumin/creatinine ratio (ACR): HR 1.22, $95 \%$ CI 1.03-1.43, $P=0.02$. In additional analyses in the 3 cohorts, the upper tertile of baseline plasma copeptin was associated with a higher ACR at the end of follow-up: $30.1 \pm 2.8$ (T1), $31.1 \pm 2.8$ (T2), and $37.3 \pm 2.8 \mathrm{mg} / \mathrm{g}$ (T3) (mean \pm SEM, ANCOVA, $P<0.0001$, same adjustments as above plus duration of follow-up).

Interaction between sex and copeptin. Plasma copeptin at baseline was higher in men than in women: $4.97 \mathrm{pmol} / 1$ (4.18) versus $3.08 \mathrm{pmol} / 1$ (2.08) for DESIR, $6.92 \mathrm{pmol} / 1$ (5.80) versus $4.21 \mathrm{pmol} / 1$ (3.52) for MDCS-CC, and $6.13 \mathrm{pmol} / 1$ (5.25) versus $3.56 \mathrm{pmol} / 1$ (3.05) for PREVEND (median and interquartile range [IQR], $P<0.0001$ for all comparisons). The associations of baseline copeptin with the outcomes during follow-up were observed both in men and women (Supplemental Tables 4 and 5), and no interaction between sex and copeptin was observed in the analyses (Figure 5). 
Table 1. Characteristics of participants at baseline by tertiles of plasma copeptin

\begin{tabular}{|c|c|c|c|c|}
\hline Copeptin tertiles & T1 & T2 & T3 & $P$ \\
\hline$n$ & 4,571 & 4,545 & 4,481 & \\
\hline \multicolumn{5}{|l|}{ Plasma copeptin (pmol/l) } \\
\hline Women & $2.06(0.77)$ & $3.51(1.04)^{A}$ & $6.45(3.14)^{A, B}$ & $<0.0001$ \\
\hline Age (yr) & $50 \pm 10$ & $50 \pm 11$ & $51 \pm 11^{A, B}$ & $<0.0001$ \\
\hline Sex: men & $2,133(46.7 \%)$ & $2,441(46.3 \%)$ & $2,078(46.4 \%)$ & 0.93 \\
\hline $\mathrm{DBP}(\mathrm{mmHg})$ & $78 \pm 10$ & $78 \pm 11$ & $79 \pm 11^{A, B}$ & $<0.0001$ \\
\hline Antihypertensive treatment & $509(11.1 \%)$ & $516(11.4 \%)$ & $576(12.9 \%)$ & 0.02 \\
\hline Use of diuretics & $135(3.0 \%)$ & $131(2.9 \%)$ & $175(3.9 \%)$ & 0.009 \\
\hline Use of ACE-I or ARBs & $147(3.2 \%)$ & $153(3.4 \%)$ & $188(4.2 \%)$ & 0.03 \\
\hline Plasma cystatin C (mg/l) & $0.85 \pm 0.14$ & $0.88 \pm 0.15^{A}$ & $0.92 \pm 0.19^{\mathrm{A}, \mathrm{B}}$ & $<0.0001$ \\
\hline FPG (mmol/l) & $5.00 \pm 0.88$ & $5.00 \pm 0.87$ & $5.13 \pm 1.28^{\mathrm{A}, \mathrm{B}}$ & $<0.0001$ \\
\hline Glycemic status: NFG/IFG/DM (\%) & $93.4 / 4.3 / 2.3$ & $93.7 / 3.9 / 2.4$ & $92.0 / 4.4 / 3.7$ & 0.0003 \\
\hline Total cholesterol (mmol/l) & $5.76 \pm 1.08$ & $5.73 \pm 1.07$ & $5.81 \pm 1.11^{\mathrm{B}}$ & 0.004 \\
\hline LDL cholesterol (mmol/l) & $3.83 \pm 0.97$ & $3.77 \pm 0.95$ & $3.79 \pm 0.99$ & 0.05 \\
\hline HDL cholesterol (mmol/l) & $1.46 \pm 0.42$ & $1.45 \pm 0.41$ & $1.45 \pm 0.44$ & 0.45 \\
\hline Triglycerides (mmol/l) & $1.26 \pm 0.81$ & $1.27 \pm 0.80$ & $1.36 \pm 1.21^{\mathrm{A}, \mathrm{B}}$ & $<0.0001$ \\
\hline \multicolumn{5}{|c|}{$\begin{array}{l}\text { Data are expressed as mean } \pm \text { SD, median (interquartile range) (ACR and copeptin), or } n(\%) \text {. Copeptin tertiles are cohort and sex specific. Statistical tests are } \\
\text { ANOVA with log-transformed data or Wilcoxon (rank-sum) test (ACR and copeptin) for qualitative parameters, and Pearson's } \chi^{2} \text { test for qualitative variables. } \\
\text { Tukey-Kramer honest significant difference test following ANOVA or Wilcoxon test was used for comparisons of each pair. }{ }^{\circledR} \text { Significantly different }(P<0.05) \\
\text { from T1; }{ }^{B} \text { significantly different }(P<0.05) \text { from T2. Plasma cystatin } C \text { was assessed only in the PREVEND cohort. ACR, albumin-to-creatinine ratio, assessed } \\
\text { only in DESIR and PREVEND cohorts; FPG, fasting plasma glucose; NFG, normal fasting glucose; IFG, impaired fasting glucose; DM, diabetes mellitus. SBP: } \\
\text { systolic blood pressure; DBP, diastolic blood pressure; ACE-I, angiotensin-converting enzyme inhibitors; ARBs, angiotensin receptor blockers. }\end{array}$} \\
\hline
\end{tabular}

\section{Discussion}

In the present investigation, in 3 large prospective community-based European cohorts, high levels of plasma copeptin at baseline were associated with an increased incidence of stage $3 \mathrm{CKD}$, with kidney function decline assessed by the KDIGO criterion "certain drop in eGFR" (2) and by the slope of eGFR over time as well as with incident microalbuminuria during follow-up. The risk of microalbuminuria was mainly driven by the highest copeptin tertile, while the risk for eGFR-based outcomes increased progressively with increasing copeptin tertiles. The associations remained significant following adjustment for kidney function and other classical risk factors for $\mathrm{CKD}$ at baseline. In this regard, the risk associated with high plasma copeptin was similar to the risk associated with high blood pressure and arterial hypertension and was higher than the risk associated with high total cholesterol (Figure 3), two well-established risk factors for CKD.

To our knowledge, this is the first study to evaluate copeptin as a marker of CKD risk, prospectively in a large number of people from the general population and included a large array of kidney outcomes. Previous reports from these cohorts dealt only with a subset of participants and/or limited outcomes $(25,26,28,30)$. Other population-based studies supporting the link between high levels of vasopressin and kidney function decline used surrogate markers of vasopressin secretion, such as fluid or plain water intake assessed by questionnaires, urine volume, or estimated urine osmolality $(7,8,31)$. Copeptin was associated with kidney length and prevalence of simple cysts in a family-based cross-sectional study of the general population (32), with other specific kidney disease diagnoses, including acute tubulointerstitial nephritis and hydronephrosis, in a population-based study (33), and with markers of disease severity, including glomerular filtration rate (GFR) and albuminuria, in patients with $\operatorname{ADPKD}(21,23)$. Plasma copeptin was also associated with the risk of severe kidney outcomes, including the doubling of plasma creatinine concentration and/or the incidence of ESRD, in people with type 1 or type 2 diabetes $(18,20)$. In the present investigation in cohorts from the general 

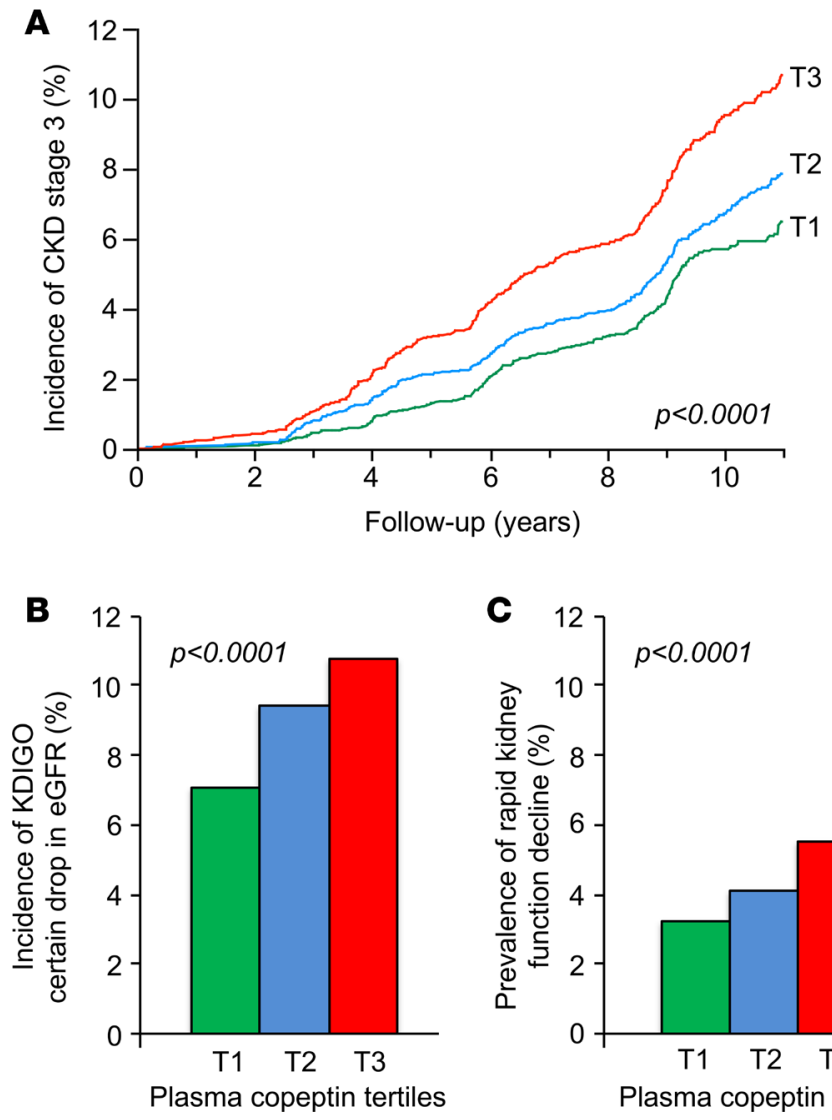

Figure 2. eGFR decline during follow-up by tertiles of plasma copeptin at baseline. (A) Kaplan-Meier curves for the incidence of stage 3 CKD, defined as eGFR $<60 \mathrm{ml} / \mathrm{min} / 1.73 \mathrm{~m}^{2}$ criterion. $\mathrm{T} 1$ ( $n=$ 4,431), T2 ( $n=4,362)$, and T3 $(n=4,240)$. (B) Incidence of CKD defined by KDIGO "certain Drop in eGFR." T1 $(n=4,571), \mathrm{T} 2(n=4,545)$, and T3 $(n=4,481)$. (C) Prevalence of rapid kidney function decline defined by an eGFR slope steeper than $-3 \mathrm{ml} / \mathrm{min} / 1.73 \mathrm{~m}^{2}$ per year. $\mathrm{T} 1(n=4,571)$, T2 $(n=4,545)$, and T3 $(n=4,481)$. Data are from pooled cohorts; logrank test $(\mathbf{A})$; Pearson's $\chi^{2}$ test (B and $\left.\mathbf{C}\right)$.

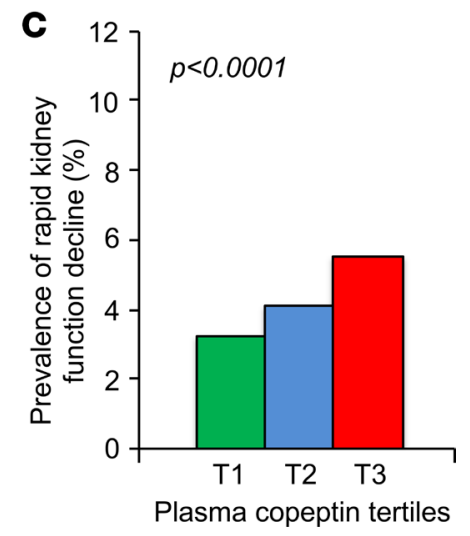

population, associations with severe kidney outcomes (eGFR slope steeper than $-5 \mathrm{ml} / \mathrm{min} / 1.73 \mathrm{~m}^{2}$ or ESRD) could not be assessed, as their prevalence or incidence during follow-up was far too low as to be studied as endpoints. A few short-term pilot studies showed that increased water intake can lead to a significant decrease in plasma copeptin concentration in healthy individuals $(34,35)$, especially in those with high plasma copeptin at baseline (34), as well as in stage $3 \mathrm{CKD}$ patients (36). However, data on kidney outcomes from intervention studies modifying vasopressin secretion or action are scarce. Administration for 36 months of tolvaptan, a V2 receptor antagonist, in patients with $\mathrm{ADPKD}$ was associated with slower kidney growth and functional decline and with a reduced frequency of ADPKD-related complications (37). An ongoing trial is now assessing the long-term efficacy and safety of increased water intake to prevent the progression of kidney growth in ADPKD patients (38). Recently, in a randomized intervention study in patients with stage $3 \mathrm{CKD}$, coaching to increase water intake compared with coaching to maintain baseline water intake did not significantly slow the decline in kidney function after 1 year (39). However, patients in the increased hydration group presented only a modest increase in 24-hour urine volume and a small decrease in plasma copeptin during follow-up. The authors concluded that the study may have been underpowered to detect a clinically important difference (39).

Higher plasma copeptin concentrations in men than in women have been consistently observed in the general population $(14,25-29)$ as well as in people with diabetic (18-20) or nondiabetic kidney disease (21, $23,24)$. Sex-related differences were reported on the association of plasma copeptin with type 2 diabetes and cardiovascular traits $(40,41)$ and on the association of allelic variations in the AVP gene with plasma copeptin (42). Nevertheless, in the present study, we observed no interaction between sex and copeptin on the associations of sex-specific tertiles or log-transformed copeptin with kidney outcomes, suggesting that the associated risk of CKD was essentially similar in men and women.

There are limitations of our study to acknowledge. We have not measured the true GFR with one of the gold-standard methods, as they are not easily applicable to large cohort studies. Instead, we used estimations based on plasma creatinine or cystatin $C$ levels. We have measured copeptin as a surrogate of vasopressin. Although their plasma concentrations correlate over a wide range of plasma and/or urine osmolalities, the strength of the correlation might vary with the GFR (14). However, the correlation seems relatively stable for eGFR $>30 \mathrm{ml} / \mathrm{min} / 1.73 \mathrm{~m}^{2}$ (43), which corresponds to all but 8 of the participants in 
Table 2. Risk of eGFR outcomes during follow-up by tertiles of plasma copeptin at baseline

\begin{tabular}{|c|c|c|c|c|c|c|}
\hline & \multicolumn{2}{|c|}{ Stage 3 CKD (eGFR < $\left.60 \mathrm{ml} / \mathrm{min} / 1.73 \mathrm{~m}^{2}\right)$} & \multicolumn{2}{|c|}{ KDIGO “Certain drop in eGFR" } & \multicolumn{2}{|c|}{ Rapid kidney function decline } \\
\hline & $\mathrm{HR}(95 \% \mathrm{Cl})$ & $P$ & OR (95\% Cl) & $P$ & OR $(95 \% \mathrm{Cl})$ & $P$ \\
\hline \multicolumn{7}{|l|}{ Model 1} \\
\hline T3 vs. T2 & $1.28(1.11-1.47)$ & 0.0005 & $1.19(1.03-1.37)$ & 0.02 & $1.25(1.02-1.54)$ & 0.03 \\
\hline T2 vs. T1 & $1.17(1.00-1.36)$ & 0.05 & $1.38(1.19-1.61)$ & $<0.0001$ & $1.43(1.14-1.79)$ & 0.002 \\
\hline $\log _{e}[$ copeptin] & $1.35(1.22-1.49)$ & $<0.0001$ & $1.42(1.28-1.56)$ & $<0.0001$ & $1.47(1.26-1.72)$ & $<0.0001$ \\
\hline T3 vs. T2 & $1.36(1.16-1.59)$ & 0.0001 & $1.23(1.05-1.43)$ & 0.01 & $1.21(0.98-1.51)$ & 0.08 \\
\hline T2 vs. T1 & $1.07(0.90-1.27)$ & 0.45 & $1.32(1.11-1.56)$ & 0.002 & $1.49(1.17-1.89)$ & 0.001 \\
\hline $\log _{e}$ [copeptin] & $1.36(1.22-1.52)$ & $<0.0001$ & $1.42(1.27-1.58)$ & $<0.0001$ & $1.47(1.26-1.72)$ & 0.0001 \\
\hline
\end{tabular}

Copeptin tertiles are cohort and sex specific. Cox regression analyses and logistic regression analyses were performed in pooled cohorts. Hazard ratio (HR) and odds ratio (OR) with $95 \% \mathrm{Cl}$ for tertiles of plasma copeptin and for 1 unit of $\log _{\mathrm{e}}$ [copeptin]. Model 1 was adjusted for cohort membership, sex, age, and eGFR at baseline, plus duration of follow-up (for KDIGO "certain drop in eGFR" and rapid kidney function decline outcomes only). Model 2 consists of model 1 plus BMI, systolic and diastolic blood pressure, fasting plasma glucose, total and HDL cholesterol and triglycerides, use of diuretics, antihypertensive drugs and angiotensin-converting enzyme inhibitors or angiotensin receptor blockers, and smoking status at baseline. The number of incident/nonincident cases during follow-up by tertiles of baseline plasma copeptin is as follows: 300/4,131 (T1), 364/3,998 (T2), and 460/3,780 (T3) for the eGFR > $60 \mathrm{ml} / \mathrm{min} / 1.73 \mathrm{~m}^{2}$ outcome; 323/4,248 (T1), 427/4,118 (T2), and 483/3,998 (T3) for KDIGO "Certain drop in eGFR" outcome; and 148/4,423 (T1), 188/4,357 (T2), and 244/4,237 (T3) for the rapid kidney function decline outcome.

our study. We studied 3 cohorts consisting predominantly of people of European descent, and our conclusion may not apply to people from other ethnic backgrounds. Finally, because of the observational design, our study does not allow any direct demonstration of a causal relationship between vasopressin and CKD.

It has been suggested that high copeptin levels observed in people with $\mathrm{CKD}$ might merely reflect a decline in GFR (44). Both vasopressin and copeptin are small-sized molecules and thus could be subjected to renal clearance. However, results from a recent investigation suggest that renal clearance is not the predominant factor in the degradation of circulating copeptin (43). Moreover, strong experimental data have been accumulated in the last decades supporting a direct causal role for vasopressin in the pathogenesis of CKD (15). Hyperfiltration was observed in rodents chronically exposed to vasopressin action $(15,45)$. Moreover, the selective vasopressin V2 receptor agonist dDAVP was shown to induce a marked increase in urinary albumin excretion both in rats and in healthy human individuals (46). Conversely, diabetes-induced hyperfiltration, albuminuria, and kidney hypertrophy were attenuated in Brattleboro rats genetically devoid of vasopressin (47) and in rodent models of diabetes treated with a vasopressin V2 receptor antagonist $(48,49)$. Consistent with these observations, decreasing vasopressin action through high water intake slowed the decline in GFR and reduced proteinuria and kidney histological damage in rodent models of CKD (16, 50-52). Glomerular hyperfiltration leads to progressive nephron damage by increasing intraglomerular hydraulic pressure (53). The mechanism by which vasopressin induces glomerular hyperfiltration and exerts its adverse effects on the kidney is not fully elucidated (15). Hyperfiltration is likely induced by a reduction in the tubuloglomerular feedback control of GFR, secondary to a reduced sodium concentration at the macula densa (15).

In conclusion, our study demonstrates that high copeptin levels are associated with the development and the progression of $\mathrm{CKD}$ in the general population. Our results argue for the relevance in human pathology of the experimental data obtained in animal models, suggesting a causal link between vasopressin and kidney disease. Together, they provide a strong basis to design future intervention studies that assess the effect of reducing vasopressin secretion, and the potential role of high water intake, in the prevention of kidney disease in the general population.

\section{Methods}

Study population. We studied 3 European population-based cohorts: DESIR from France, MDCS-CC from Sweden, and PREVEND from the Netherlands. The cohorts were selected on the basis of availability of copeptin measurement at baseline and assessment of kidney function at baseline and follow-up. Details on the design, recruitment, and procedures of the studies have been reported previously $(25,26,28,54)$. Briefly, DESIR was 
$\mathrm{HR}(95 \% \mathrm{Cl})$

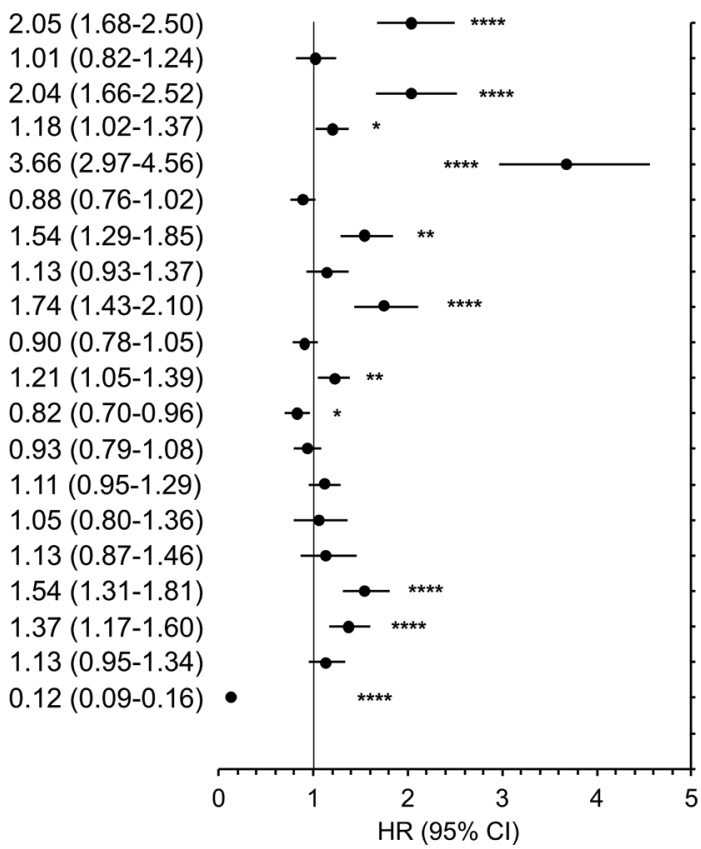

Covariates

Cohort: PREVEND vs MDCS-CC
Cohort: PREVEND vs DESIR
Cohort: DESIR vs MDCS-CC
Sex (F)
Age
BMI
Systolic BP
Diastolic BP
Antihypertensive treatment
Fasting plasma glucose
Total cholesterol
HDL cholesterol
Triglycerides
Smoking
Use of diuretics

Figure 3. Covariates associated with eGFR decline during follow-up. Left: Hazard ratio (HR) with $95 \% \mathrm{Cl}$ for baseline covariates included in Cox regression analyses (multiadjusted model) of the incidence of stage 3 CKD defined by eGFR $<60 \mathrm{ml} / \mathrm{min} / 1.73 \mathrm{~m}^{2}$ during follow-up. Right: Odds ratio (OR) with $95 \% \mathrm{Cl}$ for baseline covariates included in logistic regression analyses for KDIGO "certain drop in eGFR" criterion during follow-up. Quantitative covariates are expressed as qualitative dichotomous (below or above the median) variables, with the exception of tertiles of copeptin. eGFR, estimated glomerular filtration rate; ACE-I, angiotensin-converting enzyme inhibitor; ARBs, angiotensin receptor blockers. Data are from pooled cohorts; left, $n=13,033$; right, $n$ $=13,597 .{ }^{*} P<0.05,{ }^{* *} P<0.01,{ }^{* *} P<0.001,{ }^{* * *} P<0.0001$ by Cox regression analyses (hazard ratios) or from logistic regression analyses (odds ratio).

a 9-year prospective study conducted in 5,212 men and women (age range, 30-65 years old) from western France. The study protocol included extensive clinical and biological evaluations at inclusion (between 1994 and 1998) and at visits after 3, 6, and 9 years of follow-up. The MDCS-CC is a prospective population-based study designed to investigate the relationship between diet and other lifestyle factors on the risk of developing cancer. Between 1991 and 1996, 28,449 people living in Malmö participated in clinical examinations, which included blood sampling and a questionnaire about nutrition. After a follow-up of 16 years (between 2007 and 2012), a new clinical examination and blood sampling, including kidney function assessment, were performed in 3,700, of whom 3,186 people had complete data. Morbidity and mortality have been followed up by national registers. PREVEND is a Dutch cohort drawn from the general population (age range, 28-75 years old) from Groningen, the Netherlands. The study investigates the predictive value of urinary albumin for kidney and cardiovascular disease progression. The total screening program was completed by the 8,592 people (of the 40,856 who responded to the clinical examination invitation) who comprise the present cohort.

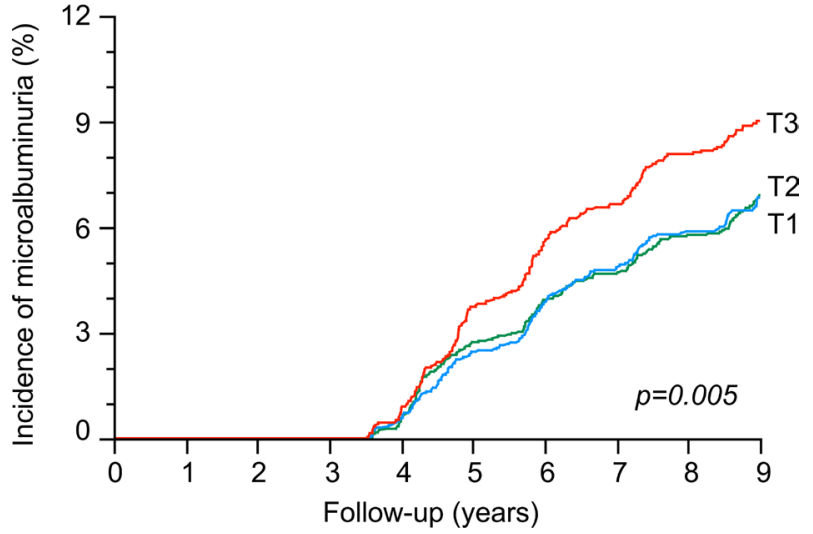

Figure 4. Kaplan-Meier curves for the incidence of microalbuminuria during follow-up by tertiles of baseline plasma copeptin. Pooled data from DESIR and PREVEND cohorts. T1 $(n=2,844), \mathrm{T} 2(n=2,812)$, and T3 $(n=2,613)$. Log-rank test. 


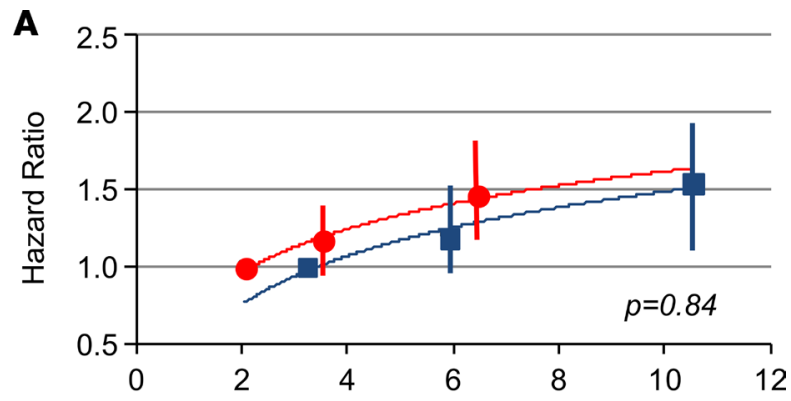

B

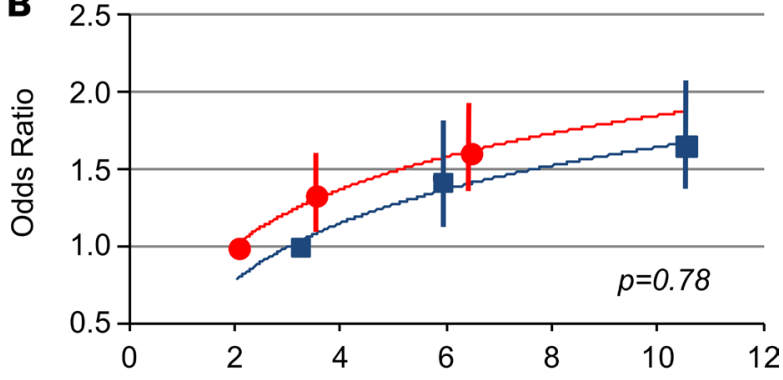

Figure 5. Hazard ratios or odds ratios with $95 \% \mathrm{Cl}$ for the association of baseline plasma copeptin with eGFR outcomes during follow-up by sex. (A) Incidence of stage 3 CKD (eGFR $<60 \mathrm{ml} / \mathrm{min} / 1.73 \mathrm{~m}^{2}$ ), (B) KDIGO "certain drop in eGFR" criterion, and (C) rapid kidney function decline. The median value for each plasma copeptin tertile is represented in blue squares for men and red circles for women. Number of participants: 6,152 men and 6,881 women (A) and 6,314 men and 7,283 women (B and $\mathbf{C}$ ). Data are from Cox proportional hazards regression analyses (A) or logistic regression analysis (B and $\mathbf{C})$, adjusted for cohort membership, sex, age, and eGFR at baseline, plus duration of follow-up (B and $\mathbf{C}$ ). The $P$ values are representative of the statistical significance for the interaction term sex/copeptin in the regression models.

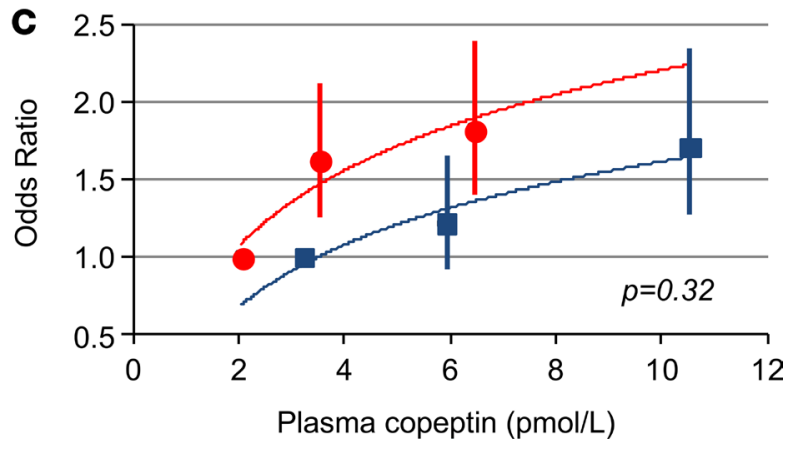

Definition of outcomes. Participants were followed for eGFR decline and new-onset of CKD during a median (IQR) duration of 9.0 (0.6), 16.5 (2.3), and 11.0 (3.9) years for DESIR, MDCS-CC, and PREVEND, respectively. We considered 4 criteria for kidney function decline and progression toward CKD during follow-up. These included, first, the incidence of stage $3 \mathrm{CKD}$ - defined as an eGFR below 60 $\mathrm{ml} / \mathrm{min} / 1.73 \mathrm{~m}^{2}$ - in at least one of the follow-up visits and, second, the "certain drop in eGFR" criterion proposed by the KDIGO group (2). GFR categories were defined as GFR $\geq 90 \mathrm{ml} / \mathrm{min} / 1.73 \mathrm{~m}^{2}$ (G1), $90>$ GFR $\geq 60 \mathrm{ml} / \mathrm{min} / 1.73 \mathrm{~m}^{2}$ (G2), $60>$ GFR $\geq 45 \mathrm{ml} / \mathrm{min} / 1.73 \mathrm{~m}^{2}$ (G3A), $45>$ GFR $\geq 30$ $\mathrm{ml} / \mathrm{min} / 1.73 \mathrm{~m}^{2}$ (G3B), $30>\mathrm{GFR} \geq 15 \mathrm{ml} / \mathrm{min} / 1.73 \mathrm{~m}^{2}$ (G4), and GFR < $15 \mathrm{ml} / \mathrm{min} / 1.73 \mathrm{~m}^{2}$ (G5). "Certain Drop in eGFR" was defined by KDIGO as a drop in GFR category accompanied by a $25 \%$ or greater drop in eGFR from baseline. As a third criterion, we considered, the slope of eGFR over time, taking into account all available (baseline and follow-up) values of eGFR for each individual. Rapid kidney function decline was defined as a slope of eGFR steeper than $-3 \mathrm{ml} / \mathrm{min} / 1.73 \mathrm{~m}^{2}$ per year. Finally, the incidence of microalbuminuria defined as ACR $\geq 30 \mathrm{mg} / \mathrm{g}$ was assessed in DESIR and PREVEND cohorts. ACR data were only available at the end of follow-up for MDCS-CC.

Laboratory procedures. Laboratory procedures for blood biochemistry assays in the DESIR, MDCSCC, and PREVEND cohorts are described elsewhere $(25,26,28,54-56)$. Copeptin concentration was measured in plasma-EDTA samples collected at baseline by an automated immunofluorescent sandwich assay (ultra-sensitive $\mathrm{B} \cdot \mathrm{R} \cdot \mathrm{A} \cdot \mathrm{H} \cdot \mathrm{M} \cdot \mathrm{S}$ Copeptin proAVP, Thermo Fischer Scientific) on the Kryptor platform $(9,10)$. The limit of detection was 0.9 pmol/1. Intra-assay CV was $<15 \%$ and $<8 \%$ for concentration ranges of $2.0-4.0 \mathrm{pmol} / 1$ and $4.0-15.0 \mathrm{pmol} / 1$, respectively. Inter-assay $\mathrm{CV}$ was $<18 \%$ and $<10 \%$, respectively, for the lower and higher copeptin concentration range (data reported by the manufacturer). Copeptin measurement in DESIR samples was performed in 2013 by Thermo Fisher Scientific or in 2015 by the authors in Paris. MDCS-CC samples were assayed locally in the authors' 
lab in Malmö in 2009. Measurement of PREVEND samples was made in 2009 by Thermo Fisher Scientific. Similar commercially available kits and equipment were used for the assay in the 3 cohorts.

Statistics. Analyses were performed in DESIR, MDCS-CC, and PREVEND pooled cohorts. Cohortand sex-specific tertiles of plasma copeptin concentration were computed to take into account cohort-related and the well-known sex-related differences in copeptin levels. eGFR was calculated using the CKDEPI study equations for serum creatinine (57) and cystatin C (58). Results are expressed as mean \pm SD, except where stated otherwise. Differences between groups were assessed by Pearson's $\chi^{2}$, Kruskal Wallis or Wilcoxon (rank-sum) tests, and ANOVA or ANCOVA. Tukey-Kramer honest significant difference test was used following ANOVA for comparisons of pairs. Kaplan-Meier curves were used to plot the incidence of CKD over time. The association of plasma copeptin with CKD was assessed by log-rank test, Cox proportional hazards survival regression analyses, or by logistic regression analyses. Adjustments for clinical and biological parameters were carried out by including these parameters as covariates in the regression models. A minimally adjusted model included as covariates cohort membership, sex, age, and eGFR at baseline, plus duration of follow-up (logistic regression analyses only). A fully adjusted model also included BMI, systolic and diastolic blood pressure, fasting plasma glucose, total and HDL cholesterol and triglycerides, use of diuretics, antihypertensive drugs and ACE inhibitors or ARBs, and smoking status at baseline. Interaction between copeptin and sex was assessed in all analyses by including in the regression model an interaction term. HR or OR, respectively, with their $95 \%$ CI were computed in these analyses. Logarithmic transformation was applied to skewed variables to fulfil the requirement of approximate normality of the residuals. Regression analyses were also made with quantitative covariates expressed as qualitative dichotomous (below or above the median) variables. Statistics used JMP (SAS Institute Inc.) and Stata (StataCorp) softwares. $P<0.05$ was considered statistically significant.

Study approval. The DESIR research protocol was approved by the ethics committee of Bicêtre Hospital, Kremlin-Bicêtre, France. MDCS-CC study protocols were approved by the regional ethics committee of Lund University, Lund, Sweden. The PREVEND study was approved by the medical ethics committee of the University of Groningen. All studies were conducted in accordance with the guidelines of the Declaration of Helsinki principles. All participants provided written informed consent.

\section{Author contributions}

REB, SJLB, RR, OM, RTG, and GV designed the study. REB, IT, EM, LMK, SE, GL, MM, BB, SJLB, RR, $\mathrm{OM}$, RTG, and GV acquired, analyzed, or interpreted data. REB, RR, and GV wrote the manuscript. IT, EM, LMK, SE, GL, KM, MM, FF, BB, NB, LB, SJLB, OM, and RTG contributed to discussion and revised and edited the manuscript. GV is the guarantor of this work and, as such, had full access to all of the data in the study and takes responsibility for the integrity of the data and the accuracy of the data analysis.

\section{Acknowledgments}

REB was supported by a Conventions Industrielles de Formation par la Recherche doctoral grant from INSERM and the Danone Research Centre for Specialized Nutrition. The DESIR study was funded by INSERM contracts with Caisse Nationale de l'Assurance Maladie des Travailleurs Salariés, Lilly, Novartis Pharma, and Sanofi-Aventis; INSERM (Réseaux en Santé Publique, Interactions entre les déterminants de la santé, Cohortes Santé TGIR 2008); the Association Diabète Risque Vasculaire; the Fédération Française de Cardiologie; La Fondation de France; Association de Langue Française pour l'Etude du Diabète et des Maladies Métaboliques/Société Francophone de Diabétologie; l'Office national interprofessionnel des vins; Ardix Medical; Bayer Diagnostics; Becton Dickinson; Cardionics; Merck Santé; Novo Nordisk; Pierre Fabre; Roche; and Topcon. The MDCS-CC study was supported by the European Research Council, the Swedish Medical Research Council, the Swedish Heart and Lung Foundation, the Medical Faculty of Lund University (Malmö), Malmö University Hospital, the Albert Pählsson Research Foundation, the Crafoord Foundation, the Ernhold Lundströms Research Foundation, the Region Skane, the Hulda and Conrad Mossfelt Foundation, the King Gustaf V and Queen Victoria Foundation, the Novo Nordisk Foundation, and the Wallenberg Foundation. Thermo Fisher Scientific and Dade-Behring provided support for assay measurements. The PREVEND study was funded by a grant from the Dutch Kidney Foundation (Nierstichting, the Netherlands). Funders and sponsors of DESIR, MDCS-CC, and PREVEND recruitment and the original studies had no role in the design and conduct of the present investigation, analysis, and interpretation of data; preparation 
or approval of the manuscript; and the decision to submit the manuscript for publication. See Supplemental Acknowledgments for DESIR, MDCS-CC, and PREVEND details.

Address correspondence to: Gilberto Velho, INSERM UMRS 1138 (Équipe 2), Centre de Recherche des Cordeliers, 15 rue de 1'École de Médecine, 75006 Paris, France. Phone: 33.1.44.27.81.14; Email: gilberto.velho@inserm.fr.

1. Levin A, et al. Global kidney health 2017 and beyond: a roadmap for closing gaps in care, research, and policy. Lancet. 2017;390(10105):1888-1917.

2. Levin A, Stevens PE. Summary of KDIGO 2012 CKD Guideline: behind the scenes, need for guidance, and a framework for moving forward. Kidney Int. 2014;85(1):49-61.

3. Clark WF, Sontrop JM, Huang SH, Moist L, Bouby N, Bankir L. Hydration and Chronic Kidney Disease Progression: A Critical Review of the Evidence. Am J Nephrol. 2016;43(4):281-292.

4. Johnson RJ, et al. Hyperosmolarity drives hypertension and CKD--water and salt revisited. Nat Rev Nephrol. 2014;10(7):415-420

5. Glaser J, et al. Climate Change and the Emergent Epidemic of CKD from Heat Stress in Rural Communities: The Case for Heat Stress Nephropathy. Clin J Am Soc Nephrol. 2016;11(8):1472-1483.

6. Wesseling C, et al. Resolving the enigma of the mesoamerican nephropathy: a research workshop summary. Am J Kidney Dis. 2014;63(3):396-404

7. Strippoli GF, Craig JC, Rochtchina E, Flood VM, Wang JJ, Mitchell P. Fluid and nutrient intake and risk of chronic kidney disease. Nephrology (Carlton). 2011;16(3):326-334.

8. Sontrop JM, et al. Association between water intake, chronic kidney disease, and cardiovascular disease: a cross-sectional analysis of NHANES data. Am J Nephrol. 2013;37(5):434-442.

9. Morgenthaler NG, Struck J, Alonso C, Bergmann A. Assay for the measurement of copeptin, a stable peptide derived from the precursor of vasopressin. Clin Chem. 2006;52(1):112-119.

10. Morgenthaler NG. Copeptin: a biomarker of cardiovascular and renal function. Congest Heart Fail. 2010;16 Suppl 1:S37-S44.

11. Heida JE, et al. Comparison of ex vivo stability of copeptin and vasopressin. Clin Chem Lab Med. 2017;55(7):984-992.

12. Szinnai G, et al. Changes in plasma copeptin, the c-terminal portion of arginine vasopressin during water deprivation and excess in healthy subjects. J Clin Endocrinol Metab. 2007;92(10):3973-3978.

13. Balanescu S, Kopp P, Gaskill MB, Morgenthaler NG, Schindler C, Rutishauser J. Correlation of plasma copeptin and vasopressin concentrations in hypo-, iso-, and hyperosmolar States. J Clin Endocrinol Metab. 2011;96(4):1046-1052.

14. Roussel R, et al. Comparison between copeptin and vasopressin in a population from the community and in people with chronic kidney disease. J Clin Endocrinol Metab. 2014;99(12):4656-4663.

15. Bankir L, Bouby N, Ritz E. Vasopressin: a novel target for the prevention and retardation of kidney disease? Nat Rev Nephrol. 2013;9(4):223-239.

16. Wang CJ, Grantham JJ, Wetmore JB. The medicinal use of water in renal disease. Kidney Int. 2013;84(1):45-53.

17. Boertien WE, et al. Copeptin, a surrogate marker for arginine vasopressin, is associated with declining glomerular filtration in patients with diabetes mellitus (ZODIAC-33). Diabetologia. 2013;56(8):1680-1688.

18. Velho G, et al. Plasma copeptin and renal outcomes in patients with type 2 diabetes and albuminuria. Diabetes Care. 2013;36(11):3639-3645.

19. Pikkemaat M, Melander O, Bengtsson Boström K. Association between copeptin and declining glomerular filtration rate in people with newly diagnosed diabetes. The Skaraborg Diabetes Register. J Diabetes Complicat. 2015;29(8):1062-1065.

20. Velho G, et al. Plasma copeptin, kidney outcomes, ischemic heart disease, and all-cause mortality in people with long-standing type 1 Diabetes. Diabetes Care. 2016;39(12):2288-2295.

21. Meijer E, et al. Copeptin, a surrogate marker of vasopressin, is associated with disease severity in autosomal dominant polycystic kidney disease. Clin J Am Soc Nephrol. 2011;6(2):361-368.

22. Boertien WE, et al. Copeptin, a surrogate marker for vasopressin, is associated with kidney function decline in subjects with autosomal dominant polycystic kidney disease. Nephrol Dial Transplant. 2012;27(11):4131-4137.

23. Boertien WE, et al. Relationship of copeptin, a surrogate marker for arginine vasopressin, with change in total kidney volume and GFR decline in autosomal dominant polycystic kidney disease: results from the CRISP cohort. Am J Kidney Dis. 2013;61(3):420-429.

24. Meijer E, et al. Copeptin, a surrogate marker of vasopressin, is associated with accelerated renal function decline in renal transplant recipients. Transplantation. 2009;88(4):561-567.

25. Roussel R, et al. Plasma Copeptin and Decline in Renal Function in a Cohort from the Community: The Prospective D.E.S.I.R. Study. Am J Nephrol. 2015;42(2):107-114.

26. Tasevska I, Enhörning S, Christensson A, Persson M, Nilsson PM, Melander O. Increased levels of copeptin, a surrogate marker of arginine vasopressin, are associated with an increased risk of chronic kidney disease in a general population. Am J Nephrol. 2016;44(1):22-28

27. Bhandari SS, Loke I, Davies JE, Squire IB, Struck J, Ng LL. Gender and renal function influence plasma levels of copeptin in healthy individuals. Clin Sci. 2009;116(3):257-263.

28. Meijer E, Bakker SJ, Halbesma N, de Jong PE, Struck J, Gansevoort RT. Copeptin, a surrogate marker of vasopressin, is associated with microalbuminuria in a large population cohort. Kidney Int. 2010;77(1):29-36.

29. Enhörning S, Struck J, Wirfält E, Hedblad B, Morgenthaler NG, Melander O. Plasma copeptin, a unifying factor behind the metabolic syndrome. J Clin Endocrinol Metab. 2011;96(7):E1065-E1072.

30. Enhörning S, et al. Copeptin, a marker of vasopressin, in abdominal obesity, diabetes and microalbuminuria: the prospective 
Malmö Diet and Cancer Study cardiovascular cohort. Int J Obes (Lond). 2013;37(4):598-603.

31. Clark WF, et al. Urine volume and change in estimated GFR in a community-based cohort study. Clin J Am Soc Nephrol. 2011;6(11):2634-2641

32. Ponte B, et al. Copeptin is associated with kidney length, renal function, and prevalence of simple cysts in a population-based study. J Am Soc Nephrol. 2015;26(6):1415-1425.

33. Enhorning S, Christensson A, Melander O. Plasma copeptin as a predictor of kidney disease [published online ahead of print February 16, 2018]. Nephrol Dial Transplant. https://doi.org/10.1093/ndt/gfy017.

34. Enhorning S, et al. Effects of hydration on plasma copeptin, glycemia and gluco-regulatory hormones: a water intervention in humans [published online ahead of print December 14, 2017]. Eur J Nutr. https://doi.org/10.1007/s00394.017.1595-8.

35. Lemetais G, Melander O, Vecchio M, Bottin JH, Enhorning S, Perrier ET. Effect of increased water intake on plasma copeptin in healthy adults [published online ahead of print June 3, 2017]. Eur J Nutr. https://doi.org/10.1007/s00394.017.1471-6.

36. Sontrop JM, et al. Effect of increased water intake on plasma copeptin in patients with chronic kidney disease: results from a pilot randomised controlled trial. BMJ Open. 2015;5(11):e008634.

37. Torres VE, et al. Tolvaptan in patients with autosomal dominant polycystic kidney disease. N Engl J Med. 2012;367(25):2407-2418.

38. Wong ATY, et al. Randomised controlled trial to determine the efficacy and safety of prescribed water intake to prevent kidney failure due to autosomal dominant polycystic kidney disease (PREVENT-ADPKD). BMJ Open. 2018;8(1):e018794.

39. Clark WF, et al. Effect of Coaching to Increase Water Intake on Kidney Function Decline in Adults With Chronic Kidney Disease: The CKD WIT Randomized Clinical Trial. JAMA. 2018;319(18):1870-1879.

40. Abbasi A, et al. Sex differences in the association between plasma copeptin and incident type 2 diabetes: the Prevention of Renal and Vascular Endstage Disease (PREVEND) study. Diabetologia. 2012;55(7):1963-1970.

41. Then C, et al. Plasma copeptin is associated with type 2 diabetes in men but not in women in the population-based KORA F4 study. Acta Diabetol. 2015;52(1):103-112.

42. Roussel R, et al. Plasma copeptin, AVP gene variants, and incidence of type 2 diabetes in a cohort from the community. J Clin Endocrinol Metab. 2016;101(6):2432-2439.

43. Ettema EM, et al. The effect of renal function and hemodialysis treatment on plasma vasopressin and copeptin levels. Kidney Int Rep. 2017;2(3):410-419.

44. Corradi V, et al. Copeptin levels and kidney function in ADPKD: case-control study. Clin Nephrol. 2016;86(9):147-153

45. Bouby N, Ahloulay M, Nsegbe E, Déchaux M, Schmitt F, Bankir L. Vasopressin increases glomerular filtration rate in conscious rats through its antidiuretic action. J Am Soc Nephrol. 1996;7(6):842-851.

46. Bardoux P, et al. Vasopressin increases urinary albumin excretion in rats and humans: involvement of $\mathrm{V} 2$ receptors and the renin-angiotensin system. Nephrol Dial Transplant. 2003;18(3):497-506.

47. Bardoux $\mathrm{P}$, et al. Vasopressin contributes to hyperfiltration, albuminuria, and renal hypertrophy in diabetes mellitus: study in vasopressin-deficient Brattleboro rats. Proc Natl Acad Sci USA. 1999;96(18):10397-10402.

48. Bardoux P, Bruneval P, Heudes D, Bouby N, Bankir L. Diabetes-induced albuminuria: role of antidiuretic hormone as revealed by chronic V2 receptor antagonism in rats. Nephrol Dial Transplant. 2003;18(9):1755-1763.

49. E1 Boustany R, et al. Antagonism of vasopressin V2 receptor improves albuminuria at the early stage of diabetic nephropathy in a mouse model of type 2 diabetes. J Diabetes Complicat. 2017;31(6):929-932.

50. Bouby N, Bachmann S, Bichet D, Bankir L. Effect of water intake on the progression of chronic renal failure in the $5 / 6$ nephrectomized rat. Am J Physiol. 1990;258(4 Pt 2):F973-F979.

51. Sugiura T, et al. High water intake ameliorates tubulointerstitial injury in rats with subtotal nephrectomy: possible role of TGF-beta. Kidney Int. 1999;55(5):1800-1810.

52. Nagao S, et al. Increased water intake decreases progression of polycystic kidney disease in the PCK rat. J Am Soc Nephrol. 2006;17(8):2220-2227.

53. Brenner BM. Nephron adaptation to renal injury or ablation. Am J Physiol. 1985;249(3 Pt 2):F324-F337.

54. Konrat C, et al. Alcohol intake and fasting insulin in French men and women. The D.E.S.I.R. Study. Diabetes Metab. 2002;28(2):116-123.

55. Rosvall M, Janzon L, Berglund G, Engström G, Hedblad B. Incident coronary events and case fatality in relation to common carotid intima-media thickness. J Intern Med. 2005;257(5):430-437.

56. Lambers Heerspink HJ, et al. Albuminuria assessed from first-morning-void urine samples versus 24-hour urine collections as a predictor of cardiovascular morbidity and mortality. Am J Epidemiol. 2008;168(8):897-905.

57. Levey AS, et al. A new equation to estimate glomerular filtration rate. Ann Intern Med. 2009;150(9):604-612.

58. Inker LA, et al. Estimating glomerular filtration rate from serum creatinine and cystatin C. NEngl J Med. 2012;367(1):20-29. 